\title{
The "Flying Intervention Team": A Novel Stroke Care Concept for Rural Areas
}

\author{
Gordian Jan Hubert $^{\mathrm{a}}$ Frank Kraus $^{\mathrm{a}} \quad$ Christian Maegerlein $^{\mathrm{b}}$ Sabine Platen $^{\mathrm{c}}$ \\ Benjamin Friedrich $^{b}$ Hans-Ulrich Kain ${ }^{d}$ Thomas Witton-Davies ${ }^{e}$ \\ Nikolai Dominik Hubert ${ }^{a}$ Claus Zimmer ${ }^{b}$ Philip M. Bath ${ }^{f}$ \\ Heinrich J. Audebert ${ }^{\mathrm{g}}{ }^{\mathrm{h}}$ Roman L. Haberl ${ }^{\mathrm{a}}$ \\ aDepartment of Neurology, TEMPiS Telemedical Stroke Center, München Klinik Harlaching, Academic Teaching

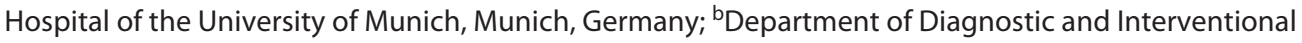 \\ Neuroradiology, Klinikum rechts der Isar, Technical University of Munich, Munich, Germany; 'Department of \\ Neurology, TEMPiS Telemedical Stroke Center, University of Regensburg, Bezirksklinikum Regensburg, Regensburg, \\ Germany; ${ }^{d}$ Community Hospital, Kliniken Kreis Mühldorf, Mühldorf, Germany; ${ }^{e}$ Department of Diagnostic and \\ Interventional Radiology and Neuroradiology, München Klinik Harlaching, Munich, Germany; ${ }^{\mathrm{f} D i v i s i o n}$ of Clinical \\ Neuroscience, Stroke Trials Unit, University of Nottingham, Nottingham, UK; ${ }^{9}$ Center for Stroke Research Berlin, \\ Charite-Universitätsmedizin Berlin, Berlin, Germany; ' Department of Neurology, Charite-Universitätsmedizin Berlin, \\ Berlin, Germany
}

\section{Keywords}

Ischemic stroke $\cdot$ Thrombectomy $\cdot$ Telemedicine $\cdot$ Health care concept $\cdot$ Helicopter

\begin{abstract}
Background: Endovascular treatment of large vessel occlusion in acute ischemic stroke patients is difficult to establish in remote areas, and time dependency of treatment effect increases the urge to develop health care concepts for this population. Summary: Current strategies include direct transportation of patients to a comprehensive stroke center (CSC) ("mothership model") or transportation to the nearest primary stroke center (PSC) and secondary transfer to the CSC ("drip-and-ship model"). Both have disadvantages. We propose the model "flying intervention team." Patients will be transported to the nearest PSC; if telemedically identified as eligible for thrombectomy, an intervention team will be
\end{abstract}

acutely transported via helicopter to the PSC and endovascular treatment will be performed on site. Patients stay at the PSC for further stroke unit care. This model was implemented at a telestroke network in Germany. Fifteen remote hospitals participated in the project, covering $14,000 \mathrm{~km}^{2}$ and a population of 2 million. All have well established telemedically supported stroke units, an angiography suite, and a helicopter pad. Processes were defined individually for each hospital and training sessions were implemented for all stroke teams. An exclusive project helicopter was installed to be available from 8 a.m. to 10 p.m. during 26 weeks per year. Key Messages: The model of the flying intervention team is likely to reduce time delays since processes will be performed in parallel, rather than consecutively, and since it is quicker to move a medical team rather than a patient. This project is currently under evaluation (clinicaltrials NCT04270513).

(c) 2021 S. Karger AG, Basel karger@karger.com www.karger.com/ced
(C) 2021 S. Karger AG, Basel 


\section{Introduction}

In most parts of the world, health care systems have been developed to deliver intravenous thrombolysis (IVT) to acute ischemic stroke patients in a timely manner. Decentralized stroke care and innovative systems, like telemedicine-assisted networks, have become increasingly popular to deliver stroke expertise to rural, sparsely populated areas in the US and Europe $[1,2]$. Telestroke systems provide special expertise for the administration of IVT via telemedicine ensuring proper selection of patients and setup of fast processes [3, 4]. Currently, $81-97 \%$ of the US population has access to IVT treatment [5].

In 2015, endovascular treatment (EVT) was shown to be effective in acute ischemic stroke patients with large vessel occlusion (LVO) [6], thus urging health care professionals to adapt their systems to this new standard of care [7-9]. EVT needs high expertise onsite, typically requires high volume activity, and cannot be delivered remotely. Nevertheless, the success of EVT regarding patient outcome is - similar to IVT - highly time dependent [10]. Rapid application of a treatment that needs skilled and well-trained interventionists is particularly challenging in non-urban areas where such expertise is rare and geographical distances are long. Therefore, new strategies are needed to provide this service with minimum delay.

In this perspective article, we provide an overview on the various current attempts to implement area-covering services and suggest a new model of care, the "flying intervention team" (FIT)-model in which the challenges of providing fast treatment (which usually means short distance to the patients' home) and high expertise (which usually means metropolitan area) can be met for remote, underserved areas. A project setup within the telestroke network Southeast Bavaria (TEMPiS) will exemplify this new model.

\section{Systems of Hyperacute Stroke Care in Rural Areas}

In order to fulfill the necessities of modern hyperacute stroke treatment, a system of care should provide access to the following:

The most frequently used strategies are the so-called "mothership" and the "drip-and-ship" approaches which have both advantages and disadvantages regarding the above mentioned necessities.

\section{The Mothership Model}

In the mothership approach, patients with an increased likelihood of LVO are identified in the commu- nity and transported directly to a comprehensive stroke center (CSC), bypassing 1 or more primary stroke centers (PSCs). As a time-consuming stopover in a PSC and secondary transfer is avoided, EVT can be initiated with relatively short delays. In addition, the CSC intervention team can perform EVT with the high experience gained by high treatment volumes $[11,12]$. A disadvantage of the mothership approach is that longer transport times to a CSC delay treatment with IVT, especially important if patients turn out not to be eligible for EVT, for example, if no proximal vessel is occluded [13]. In addition, hyperacute treatment of patients with intracranial hemorrhage is delayed and the long-distance transports of a high number of patients to a CSC may lead to a critical shortage of emergency vehicles and paramedics in the original catchment area. Last, CSCs will inevitably have to care for patients who turn out not to be eligible for EVT.

\section{The Drip-and-Ship Model}

In the drip-and-ship approach, patients are generally transported to the closest PSC and receive IVT onsite; if EVT is indicated, secondary transfer to the CSC is initiated $[8,9]$. The main advantage of this system is the timely delivery of IVT. This is advantageous when considering that more patients are eligible for IVT than for EVT (approx. 20 vs. 7\%, respectively, of all ischemic stroke patients) $[14,15]$. Also, the selection of EVT candidates is highly accurate as identification in PSC is done on the basis of computed tomography (CT) and CT-angiography (CT-A) and (tele-)expert examination. A disadvantage is the delayed start of EVT in comparison to the mothership approach. Secondary transfers of patients with severe stroke are often time consuming due to limited transport vehicle availability, multiple handovers, necessity of transport preparation, travel distances, and admittance procedures at the CSC. The drip-and-ship approach is considered favorable with increasing distance between the CSC and PSC [12, 16-19].

The mothership and drip-and-ship strategies are currently under investigation, and mathematical models are used to simulate the impact of different parameters on good outcome (e.g., distance between PSC and CSC) [12, $16,20]$. However, as real-world data are not yet available, comparisons of these and newer systems are urgently needed.

Onsite EVT services provided in PSC would be a potential solution because they promise fast onset of stroke symptoms to groin puncture. However, given the expected low absolute numbers of EVT in these hospitals, a fulltime on-site service would neither be cost-efficient nor 

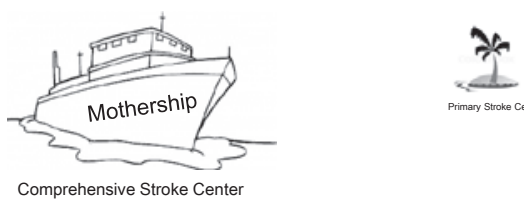

Comprehensive Stroke Center
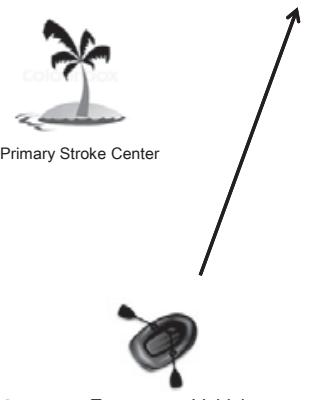

Emergency Vehicle

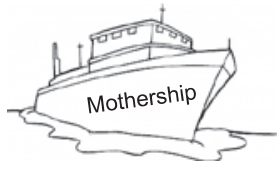

Comprehensive Stroke Center

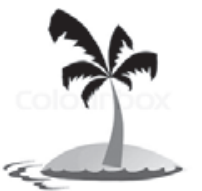

Primary Stroke Center

a

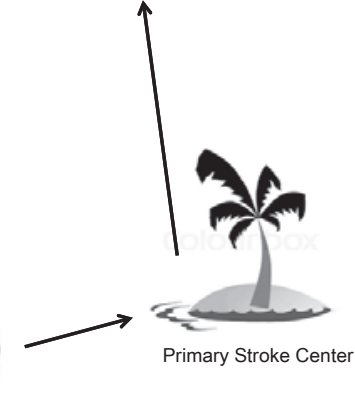

b

Emergency Vehicle

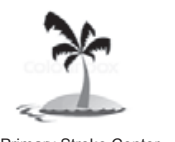

Primary Stroke Cente
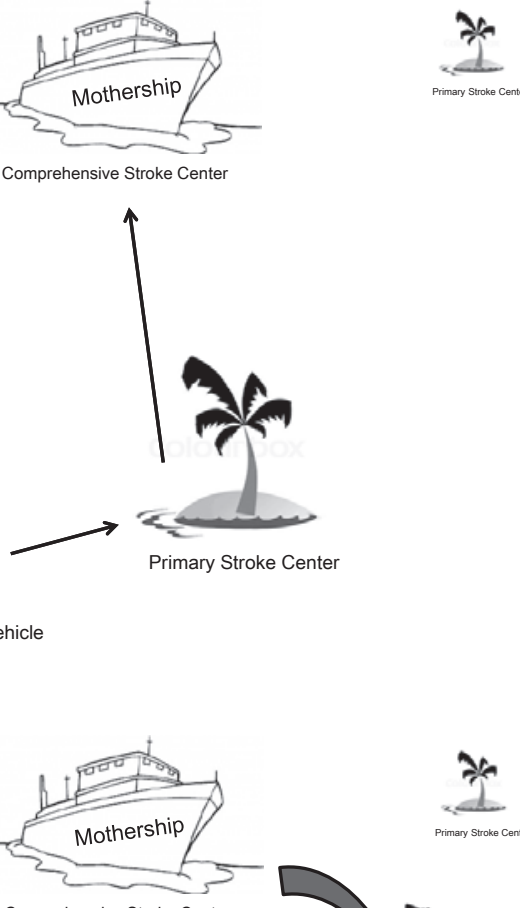

Comprehensive Stroke Center
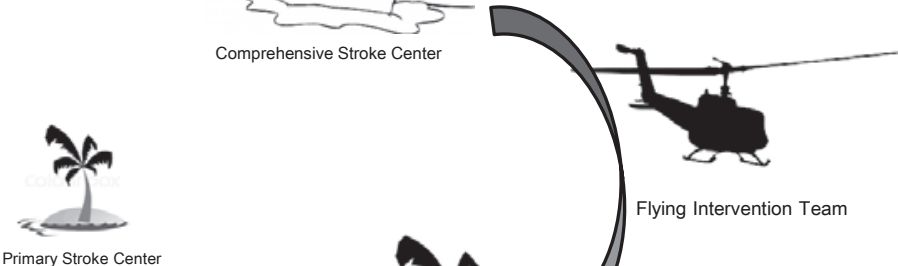

Fig. 1. Models of hyperacute stroke care: mothership (a), drip-and-ship (b), and FIT model (c). FIT, Flying Intervention Team; CSC, comprehensive stroke center; PSC, primary stroke center. 
Fig. 2. Map of FIT hospitals within the telestroke network TEMPiS; stars = TEMPiS-PSC included in the FIT project; gray dots = TEMPiS-PSC not participating in the FIT-project; $\mathrm{H}$, helicopter base ( $\mathrm{Mu}$ nich Harlaching, Klinikum rechts der Isar); black dot, network center (Regensburg). PSC, primary stroke center; FIT, flying intervention team.

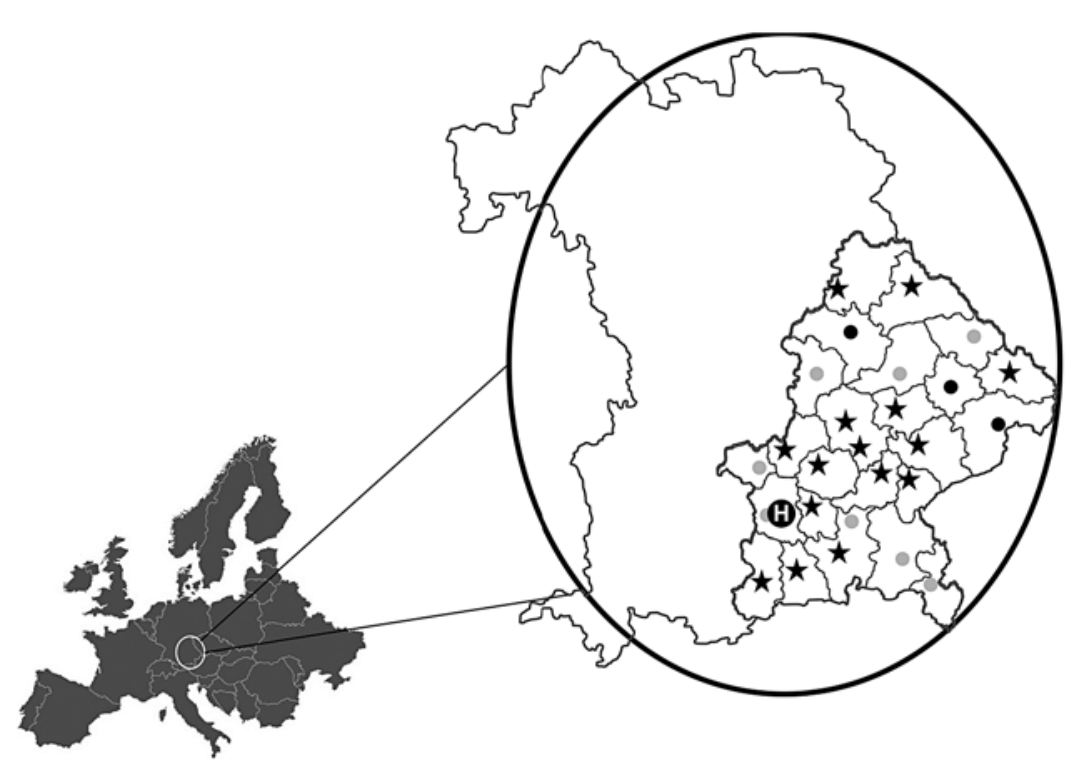

will interventional practitioners have sufficient practice to retain competency. A possible way to overcome this dilemma is to transport the specialist from the CSC to the patient in the PSC.

\section{The "FIT" Model}

A team of interventionists is located centrally (e.g., at a CSC), and a dedicated helicopter service is available. In case a patient in a PSC is telemedically identified as a candidate for EVT, the interventionist and his/her assistant are flown directly to the patient at the PSC (see Fig. 1). The EVT can be performed in the local angiography suite with onsite support (anesthetist and nurse). Post-procedural care is then provided in the local stroke unit or intensive care unit of the PSC with telemedicine support. This model fulfills all requirements for hyperacute ischemic stroke care: rapid application of IVT (in the PSC close to patient's home), rapid application of EVT (in the PSC close to patient's home), and high expertise (staff from CSC).

\section{The FIT Setup in the Telestroke Network TEMPiS}

The setup of the novel stroke care system was embedded in the existing TEMPiS network that consists of 2 CSC hubs and 24 PSC spoke hospitals. The hub hospitals have dedicated (tele)stroke units with specialized staff and have experience in treating stroke patients including severe strokes between 6 and 17 years (SD). The hubs provide telemedical consultation via bidirectional videoconference and imaging transfer for all hospitals.

Fifteen of these PSC telestroke units are eligible for the new model (see Fig. 2). The other 9 hospitals were not included because they had no angiography suite onsite, preferred to transfer their patients to a specific partner hospital, did not have enough experience in stroke care to be included, or were developing plans to set up a team of neurointerventionists themselves. These 15 PSCs cover a region of $14,000 \mathrm{~km}^{2}$ and a population of 2 million [21]. Mean distance between spoke hospitals and the nearest hub is $71 \mathrm{~km}$ (minimum $30 \mathrm{~km}$, maximum $147 \mathrm{~km}$ ). All districts covered by spokes are considered rural or intermediately populated areas according to NUTS3 criteria [22]. All these PSC have an adequate angiography room and adequate stroke care quality. In all hospitals, a helicopter pad is on site in foot distance, but for 1 , where an additional transfer of the team with a conventional car is required (approximately $1.5 \mathrm{~km}$ distance).

Two CSCs in Munich (Klinikum rechts der Isar, München Klinik Harlaching) set up an intervention team consisting of 5 neurointerventionists and 6 angiography nurses. When on call for the network, they are free from all other time critical duties and are not covering for patient care in the hub hospitals. A helicopter service is reserved for the EVT service in the network covering from 8 a.m. to 10 p.m. Although this helicopter is not part of the regular emergency service in Bavaria/Germany, German 


\section{Secondary transfer to CSC}

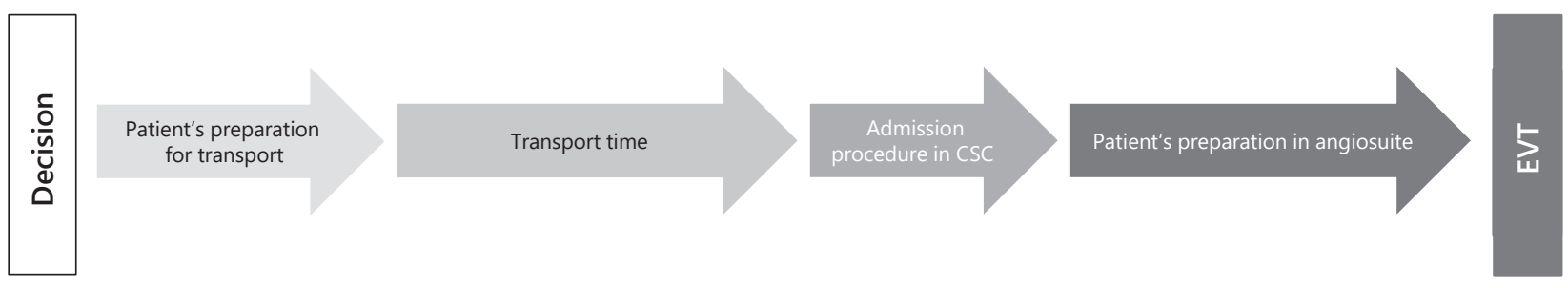

\section{TEMPiS Flying Intervention team}

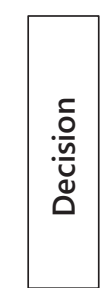

Transport time

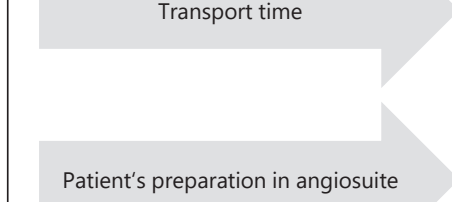

Patient's preparation in angiosuite

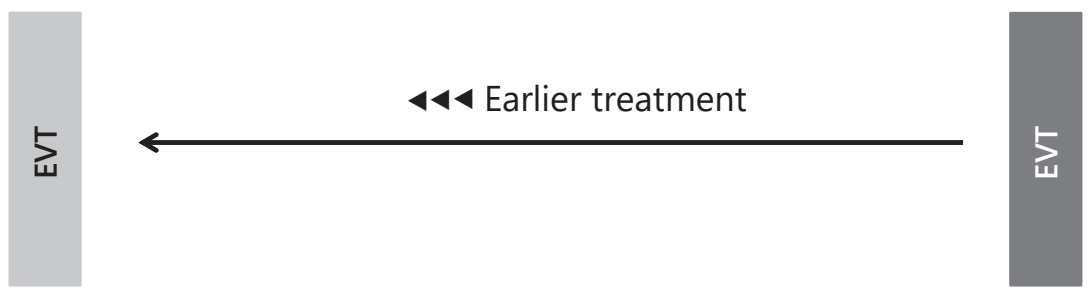

Fig. 3. Processing depending on model: sequential processing in drip-and-ship model (a); parallel processing in FIT model (b). CSC, comprehensive stroke center; EVT, endovascular treatment; FIT, flying intervention team; TEMPiS, telestroke network Southeast Bavaria, Germany.

Federation Aviation Office has approved it as Helicopter Emergency Medical Service (HEMS as per EU regulation 956-2012). This includes allowance to fly in lower weather conditions than private helicopter flight operations, and the possibility to cross controlled aviation zones and to use helicopter pads in all participating PSC.

Hospital protocols have been developed individually for each PSC, covering all processes of EVT. Each intervention team is familiar with the surroundings in PSC and has been trained on the local angiography suite beforehand. Onsite or on-call anesthetic and technical assistance from the PSC cover for these emergencies.

Special materials such as endovascular catheters and stent retrievers are stored at the center and carried by the intervention team with each deployment to the intervention site. Basic necessary material is packed as kits and stored in local hospitals for preparation already before arrival of the intervention team.

In case of a stroke patient admitted to a local hospital, the teleconsultant is contacted immediately via phone be-

The Flying Intervention Team fore CCT. If likelihood of LVO is high (G-FAST-Score $\geq 3$ ), the teleconsultant sets off a pre-alarm to the intervention team and helicopter crew in order to prepare for possible takeoff. After videoconference and reading of the CT scan and CT-A, decision on EVT is made and the final alarm initiated. The helicopter base is located at one of the 2 CSCs (Munich Harlaching). In case, the team of the same CSC is on call, the flight will start directly at this hospital, and in case the team of the second CSC is on call, the helicopter will fly to their hospital ( $3 \mathrm{~min}$ flight time) and will pick up the crew there. The intervention team is flown with special material to PSC. During the time of the flight, the patient is already prepared in the angiography room of the PSC. Post-procedural care is carried out by the local team of PSC's stroke unit or ICU with the possibility of instant telemedical review by the network center.

The FIT service is currently provided for 26 weeks/ year. During all other weeks, the conventional drip-andship-model is in place. This approach will allow close eval- 
uation of the novel system regarding time delay, clinical outcome, and cost efficiency (clinicaltrials NCT04270513). In the participating PSC, 200 EVTs are expected per year.

\section{Potential Advantages and Hazards}

\section{Time Reductions by Parallel Processing}

In both the mothership and particularly the drip-andship model, the various steps of stroke workup and treatment are mainly carried out in a sequential manner. Time delays from decision to EVT in the drip-and-ship model can be split up in to 4 parts: (i) organization of EMS (emergency medical service) vehicle and preparation of a patient for transport, (ii) transport time, (iii) admission of a patient at a CSC (recheck of indication, possibly repeated imaging, etc.), and (iv) preparation of a patient in an angiography room. In the FIT model, the delays (i) and (iii) do not exist since the patient is not transferred. And preparation of the patient in the angiography room can be initiated right after decision for EVT and conducted in parallel while the intervention team is flying in (see Fig. 3). Once the team arrives, groin puncture can be performed without further delays.

\section{Further Potential Advantages}

A report from drip-and-ship cohorts showed that ambulance transportation of patients during or shortly after IVT is associated with slightly increased mortality, intracranial hemorrhage, and other IVT-related complications [23]. The specific effect of transportation in helicopters on an ischemic brain has not been thoroughly investigated as it involves unusual physical factors such as vibration, noise, accelerations, and rapid changes in barometric pressure and partial pressure of oxygen [24].

Airborne transport of a medical team is usually initiated much faster than that of patients and requires a less sophisticated helicopter, while at the same time transport associated medical hazards of patients is avoided. Patients can stay in their local PSC with all advantages for relatives, discharge management, and organization of stroke aftercare. By basing the decision on EVT on CT-A and perfusion in the PSC, unnecessary transportation of false positive patients and overcrowding of CSC emergency departments can be diminished, thus preventing possible adverse impact on patients' outcome [25].

In case of pandemic, such as COVID-19 in 2020, this system potentially reduces spreading of disease. The primary admission of a patient in a PSC and secondary transfer to a CSC means involvement of 2 EMS teams and
2 in-hospital teams. In the FIT-model, the second EMS team is not involved and only the interventionalist and the assistance are in contact with the patient (compared to a full second stroke team). Of course, the risk of disease transmission is not zero. There is a potential hazard that the FI team can become infected or spread the disease in the local hospital. However, the risk is smaller because less people are involved and infection status is usually more monitored in medical staff than in acute patients.

\section{Potential Drawbacks}

First, in cases of spontaneous recanalization by IVT, the transport of the neurointervention team may be in vain, causing relevant costs. Second, more complex causes of LVO such as wide dissections or difficult access conditions may be challenging to treat with somewhat limited neurointerventional equipment and may restrain the use of the FIT. Third, some periprocedural complications, such as large artery rupture, how rare they may be, may not be treatable at a PSC, thus requiring emergency secondary transfer.

Fourth, helicopters cannot fly under all weather conditions. These situations can be minimized by the presence of 2 instrument rated pilots with night ratings. But there will always have to be a backup system such as acute transfers to a CSC.

Fifth, if the FIT is deployed, new incoming thrombectomy cases within the network will need to be transferred for treatment. And last, the requirements for the participating PSCs are high, demanding excellent quality of stroke care and dedicated staff. This may limit the wide implementation of this system.

\section{Open Questions}

Whether EVT and post-procedural care is effective and safe in PSC needs to be evaluated by closely monitoring recanalization rates, complications, patient outcomes, and other safety parameters. In PSCs, angiography is more often monoplanar, potentially restraining the procedure; however, 1 report suggested that monoplanar-based EVT can be performed with equal success, slightly faster, and with less radiation compared to using biplanar equipment [26]. It is also unclear whether anesthesia teams, not highly experienced with the care of complex cerebrovascular patients and the nuanced blood pressure management, have a hazardous effect on patients' outcome. Only PSC with longstanding experience in stroke care and established stroke units should be considered eligible for the FIT model. But some aspects of post-procedural care of EVT patients are not as well established here as in a CSC. Whether intense training and growing experience of PSC 
personnel can resolve these challenges needs to be investigated. Several groups described the use of a car/metro to drive a neurointerventionist to external hospitals [27-29]. These mobile teams lead to reduction in time delay until treatment in all studies [27-29]. Two publications reported equal or slightly improved neurological outcome at discharge in these patients compared to patients having been transferred for treatment $[27,29]$. One field report was published using the strategy to fly a neurointerventionist to a PSC in an individual case [30]. However, stroke unit care (and/or intensive care) performed by well-trained, dedicated staff is essential for post-procedural care and will be one of the indispensable requirements of the PSC.

Cost-effectiveness also needs to be evaluated. Ongoing costs of this system are high as the helicopter, pilot, and the intervention service have to be provided. On the other hand, the setup of several local intervention teams throughout the country as an alternative system would also be associated with substantial financial costs. Some immediate costs savings will be found in reduced transfers (e.g., shorter primary transfers and fewer secondary transfers and fewer tertiary transfers back home). Moreover, if reduction of time to treatment leads to better outcome, long-term costs can be reduced.

\section{Conclusion}

Hyperacute stroke systems of care need to provide fast IVT, fast EVT, and access to high expertise. Current concepts include the "mothership model" and the "drip-andship model." Neither model fully accomplishes the needs of a perfect system of care. While the mothership model cannot provide fast IVT for patients with longer distance to a CSC, the drip-and-ship model cannot provide fast EVT.

The "FIT" combines the advantages of both approaches. It delivers fast IVT by driving the patient to the closest PSC, it delivers fast EVT by rapidly flying the intervention team to PSC, and it assures high neurological and neurointerventional expertise is provided by a high-volume CSC team over all parts of the process. Whether the FIT model is faster, effective, and safe for patients is under evaluation.

\section{Acknowledgements}

The authors thank all participating centers for their tremendous energy and contribution in setting this project up. The authors also thank Felix Schlachetzki (University of Regensburg, Germany) for his participation in the project and his review of the manuscript.

\section{Conflict of Interest Statement}

G.J.H., F.K., N.H. and C.M. received funding from the Bavarian health insurances for the setup of the TEMPiS-FIT project. G.J.H. received a grant from the Bavarian Ministry of Health and the Björn-Steiger-Foundation for the evaluation of the project. All other authors report no financial conflict of interest.

\section{Funding Sources}

The project was funded by Bavarian Health Insurances, the Bavarian Ministry of Health, and the Björn-Steiger-Stiftung.

\section{Author Contributions}

G.H.: drafting of first manuscript; G.H., F.K., C.M., H.U.K., N.H., and H.J.A., R.L.H.: concept design; all: contribution to concept setup, critical revision, and final approval of manuscript.

\section{References}

1 Müller-Barna P, Hubert GJ, Boy S, Bogdahn $\mathrm{U}$, Wiedmann S, Heuschmann PU, et al. TeleStroke units serving as a model of care in rural areas: 10-year experience of the TeleMedical project for integrative stroke care. Stroke. 2014;45(9):2739-44.

2 Silva GS, Farrell S, Shandra E, Viswanathan A, Schwamm LH. The status of telestroke in the United States: a survey of currently active stroke telemedicine programs. Stroke. 2012; 43:2078-85.

3 Audebert HJ, Kukla C, Clarmann von Claranau S, Kühn J, Vatankhah B, Schenkel J, et al. Telemedicine for safe and extended use of thrombolysis in stroke: the telemedic pilot project for integrative stroke care (TEMPiS) in Bavaria. Stroke. 2005;36(2):287-91.
4 Hubert GJ, Meretoja A, Audebert HJ, Tatlisumak T, Zeman F, Boy S, et al. Stroke thrombolysis in a centralized and a decentralized system (Helsinki and telemedical project for integrative stroke care network). Stroke. 2016; 47(12):2999-3004.

5 Adeoye O, Albright KC, Carr BG, Wolff C, Mullen MT, Abruzzo T, et al. Geographic access to acute stroke care in the United States. Stroke. 2014;45(10):3019-24.

6 Goyal M, Menon BK, van Zwam WH, Dippel DW, Mitchell PJ, Demchuk AM, et al. Endovascular thrombectomy after largevessel ischaemic stroke: a meta-analysis of individual patient data from five randomised trials. Lancet. 2016;387(10029): 1723-31.
7 Fisher M, Wakhloo A. Dawning of a new era for acute stroke therapy. Stroke. 2015;46(6): $1438-9$.

8 Smith EE, Schwamm LH. Endovascular clot retrieval therapy: implications for the organization of stroke systems of care in North America. Stroke. 2015;46(6):1462-7.

9 Tatlisumak T. Implication of the recent positive endovascular intervention trials for organizing acute stroke care: European perspective. Stroke. 2015;46(6):1468-73.

10 Saver JL, Goyal M, van der Lugt A, Menon BK, Majoie CB, Dippel DW, et al. Time to treatment with endovascular thrombectomy and outcomes from ischemic stroke: a meta-analysis. JAMA. 2016;316(12):127988. 
11 Davis SM, Campbell BCV, Donnan GA. Endovascular thrombectomy and stroke physicians: equity, access, and standards. Stroke. 2017;48(8):2042-4.

12 Milne MSW, Holodinsky JK, Hill MD, Nygren A, Qiu C, Goyal M, et al. Drip 'n ship versus mothership for endovascular treatment: modeling the best transportation options for optimal outcomes. Stroke. 2017;48: 791-4.

13 Fuentes B, Alonso de Leciñana M, XiménezCarrillo A, Martínez-Sánchez P, Cruz-Culebras A, Zapata-Wainberg G, et al. Futile interhospital transfer for endovascular treatment in acute ischemic stroke: the madrid stroke network experience. Stroke. 2015;46(8): 2156-61.

14 Ng FC, Low E, Andrew E, Smith K, Campbell BCV, Hand PJ, et al. Deconstruction of interhospital transfer workflow in large vessel occlusion: real-world data in the thrombectomy era. Stroke. 2017;48(7):1976-9.

15 Chia NH, Leyden JM, Newbury J, Jannes J, Kleinig TJ. Determining the number of ischemic strokes potentially eligible for endovascular thrombectomy: a population-based study. Stroke. 2016;47(5):1377-80.

16 Holodinsky JK, Williamson TS, Kamal N, Mayank D, Hill MD, Goyal M. Drip and ship versus direct to comprehensive stroke center: conditional probability modeling. Stroke. 2017;48:233-8.

17 Higashida R, Alberts MJ, Alexander DN, Crocco TJ, Demaerschalk BM, Derdeyn CP, et al. Interactions within stroke systems of care: a policy statement from the American heart association/American stroke association. Stroke. 2013;44(10):2961-84.
18 Holodinsky JK, Williamson TS, Demchuk AM, Zhao H, Zhu L, Francis MJ, et al. Modeling stroke patient transport for all patients with suspected large-vessel occlusion. JAMA Neurol. 2018;75(12):1477-86.

19 Regenhardt RW, Mecca AP, Flavin SA, Boulouis G, Lauer A, Zachrison KS, et al. Delays in the air or ground transfer of patients for endovascular thrombectomy. Stroke. 2018; 49(6):1419-25.

20 Schlemm E, Ebinger M, Nolte CH, Endres M, Schlemm L. Optimal transport destination for ischemic stroke patients with unknown vessel status: use of prehospital triage scores. Stroke. 2017;48(8):2184-91.

21 Kreisfreie Städte und Landkreise nach Fläche, Bevölkerung und Bevölkerungsdichte am 31.12.2019. Statistisches Bundesamt. https:// www.destatis.de/DE/Themen/Laender-Regionen/Regionales/Gemeindeverzeichnis/ Administrativ/04-kreise.html Accessed 9 Sep 2020.

22 Eurostat regional yearbook 2018. https:// ec.europa.eu/eurostat/statistical-atlas/gis/vie wer/?ch=C14,C06,C04,C09,TRC,TYP\& mids $=$ BKGCNT,BKGCRL,BKGPGR,C99M01,C NTOVL\&o $=1,0.5,1,1,0.7 \&$ center $=48.95158,1$ $0.75006,6 \&$ lcis $=$ C99M01\&\# Accessed 2020 Sep 9.

23 Sheth KN, Smith EE, Grau-Sepulveda MV, Kleindorfer D, Fonarow GC, Schwamm LH. Drip and ship thrombolytic therapy for acute ischemic stroke: use, temporal trends, and outcomes. Stroke. 2015;46(3):732-9.
24 Leira EC, Stilley JD, Schnell T, Audebert HJ, Adams HP. Helicopter transportation in the era of thrombectomy: the next frontier for acute stroke treatment and research. Eur Stroke J. 2016;1(3):171-9.

25 Olshaker JS. Managing emergency department overcrowding. Emerg Med Clin North Am. 2009 Nov;27(4):593-603.

26 Friedrich B, Maegerlein C, Lobsien D, Mönch S, Berndt M, Hedderich D, et al. Endovascular stroke treatment on single-plane versus. biplane angiography suites : technical considerations and evaluation of treatment success. Clin Neuroradiol. 2019 Jun;29(2):303-9.

27 Wei D, Oxley TJ, Nistal DA, Mascitelli JR, Wilson N, Stein L, et al. Mobile interventional stroke teams lead to faster treatment times for thrombectomy in large vessel occlusion. Stroke. 2017;48:3295-300.

28 Brekenfeld C, Goebell E, Schmidt H, Henningsen $\mathrm{H}$, Kraemer C, Tebben J, et al. 'Dripand-drive': shipping the neurointerventionalist to provide mechanical thrombectomy in primary stroke centers. J Neurointerv Surg. 2018;10:932-6.

29 Seker F, Möhlenbruch MA, Nagel S, Ulfert C, Schönenberger S, Pfaff J, et al. Clinical results of a new concept of neurothrombectomy coverage at a remote hospital-"drive the doctor". Int J Stroke. 2018;13(7):696-9.

30 Hui FK, El Mekabaty A, Schultz J, Hong K, Horton K, Urrutia V, et al. Helistroke: neurointerventionalist helicopter transport for interventional stroke treatment: proof of concept and rationale. J Neurointerv Surg. 2018; $10(3): 225-8$. 